\title{
An Overview of the Mechanics of Oscillating Mechanisms
}

\author{
Christopher G. Provatidis* \\ Department of Mechanical Engineering, National Technical University of Athens, Greece \\ *Corresponding author: cprovat@ central.ntua.gr \\ Received December 31, 2012; Revised April 26, 2013; Accepted May 09, 2013
}

\begin{abstract}
This paper extends a previous study on the mechanics of oscillating mechanisms in which motion of an object is produced by attached rotating eccentric masses. In addition to the well known twin contra-rotating pair (Dean drive), the single eccentric mechanism is studied. In contrast to the contra-rotating systems where the initially still object moves along a vertical track, this study shows that in case of a single eccentric mass the track of the same object is extended in both the horizontal and vertical directions. In both configurations, the path is mainly influenced by the initial linear and angular momentum of the eccentrics. While contra-rotation requires a motor to ensure constant angular velocity, in single systems the latter event is conditionally achieved per se. In order to demonstrate the significance of the initial angular momentum in the motion of the object, the two types of eccentrics were applied for the conditions of the elementary Rutherford-Bohr's model of a hydrogen atom and also of a virtual hydrogen molecule. It was found that, if intermolecular forces suddenly disappear at a specific synchronization, the virtual molecule or atom is predicted to reach the incredible altitude of $72 \mathrm{~km}$.
\end{abstract}

Keywords: Two-body motion, synchronization, inertial propulsion, dean drive, hydrogen atom

\section{Introduction}

Inertial propulsion appears in nature, mainly for navigation in diptera [1], and in industrial applications like shakers in mobile phones, wash-machines and many others [2].

The possibility to convert rotary motion into unidirectional motion has been presented by Dean [3] in mid-fifties but only recently details have been reported for the particular case of twin contra-rotating eccentrics attached to the object we wish to move $[4,5]$. The main conclusion was that motion is controlled mainly by the initial position $\phi_{0}$ of the rotating masses when the object is left to fall down into the air, and also by the magnitude of the lumped masses $m$ as well as by the angular velocity $\omega$ and the radius $r$ (the product $m \omega r$ constitutes the "angular momentum" of the propulsion system). When the motor keeps on working, the angular velocity may be controlled to be preserved at a constant value, while when it is switched off the conservation of mechanical energy demands the periodical variation of the angular velocity [5].

In addition to the abovementioned twin eccentrics, there are many cases where only one eccentric mass is attached to an object. Typical examples are: twin mechanisms suddenly broken, shakers in mobiles where the friction plays a significant role, athletes who rotate their arms during long jump, electrons that rotate around protons in atoms, and many others.

This paper aims at studying primarily the single eccentric mechanism and also to compare it with the twin one.

\section{Inertial Propulsion Mechanisms}

\subsection{Contra-rotation (Twin Eccentricity)}

\subsubsection{General Formulation}

The particular case of contra-rotating and synchronized eccentric masses has been previously investigated [4,5].

We consider the particular mechanism that consists of a horizontal object (rigid plate) characterized by a large mass $M$, on which two rigid rods of equal length (radius $r$ ) are symmetrically articulated at their one end while a smaller mass $m$ is attached to their other ends, as shown in Figure 1.

Details are given elsewhere [5], but for reasons of completeness we must make clear that:

- The object of mass $M$ is rigid and has a uniform shape in the horizontal $x$-direction.

- The eccentric masses (No.1 and No.2) rotate at equal and opposite angular velocities, $\omega(t)=\dot{\phi}(t)$ : contrarotation.

- Both masses (No. 1 and No. 2) possess the same vertical $z$-level.

- The object is left free to fall down when the orientation of the rods form an angle $\phi_{0}$ with the horizontal line, as shown in Figure 1.

Obviously, the above ideal symmetric conditions lead to an ideal upward translational motion of the mechanism.

The analysis below is based on the use of Lagrange equations. 


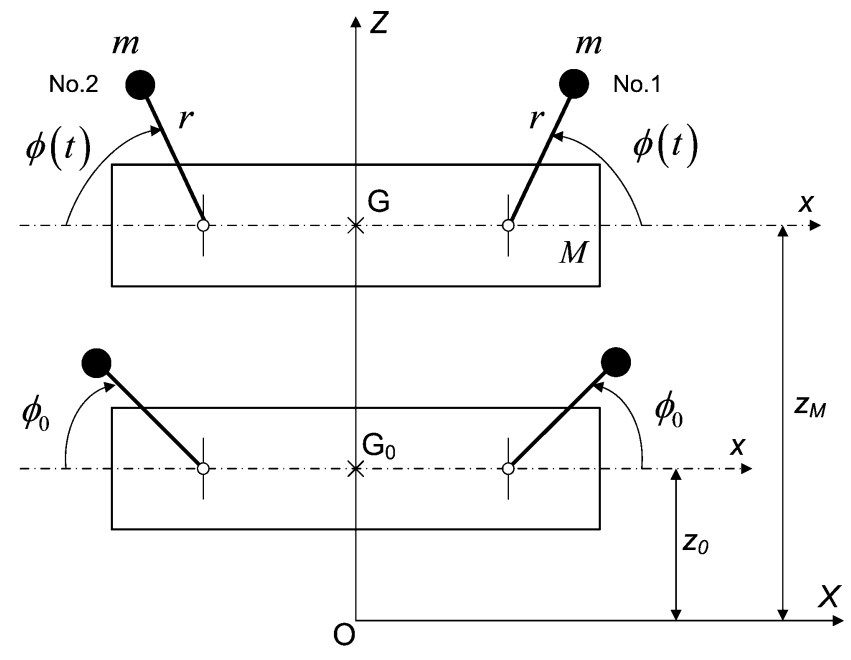

Figure 1. A typical "Dean drive", in which the centroid of the object (of mass $M$ ) moves upwards from the initial position $G_{0}$ to the current $G$

The position of the mass $M$ in the mechanism is described by the altitude $z_{M}$, whereas the position of each mass $m$ (No.1 and No.2) is determined by $z_{m}$. According to Figure 1, the relationship between them is:

$$
z_{m}=z_{M}+r \sin \phi
$$

By virtue of (1), at every time instance the velocity components of the $i$-th eccentric mass will be:

$$
\dot{z}_{m, i}=\dot{z}_{M}+r\left(\sin \phi_{i}\right)^{\bullet}=\dot{z}_{M}+r \omega_{i} \cos \phi_{i}, i=1,2
$$

and

$$
\dot{x}_{m, i}=\dot{x}_{M}+r\left(\cos \phi_{i}\right)^{\bullet}=\dot{x}_{M}-r \omega_{i} \sin \phi_{i}, i=1,2
$$

Obviously, the object does not move horizontally, that is:

$$
x_{M} \equiv 0
$$

Therefore, due to the symmetric arrangement of the two rotating masses so as, with respect to the inertial reference frame (fixed to the ground), both velocities have the same measure given by $\left|\mathbf{v}_{m}\right|=\left(\dot{x}_{m}^{2}+\dot{z}_{m}^{2}\right)^{1 / 2}$, the kinetic energy of the entire mechanism will be:

$$
\begin{aligned}
& E_{k i n}=\frac{1}{2}\left[M \dot{z}_{M}^{2}+2 m\left|\mathbf{v}_{m}\right|^{2}\right]=\frac{1}{2}\left[M \dot{z}_{M}^{2}+2 m\left(\dot{x}_{m}^{2}+\dot{z}_{m}^{2}\right)\right] \\
& =\frac{1}{2}\left\{M \dot{z}_{M}^{2}+2 m\left[(r \omega \sin \phi)^{2}+\left(\dot{z}_{M}+r \omega \cos \phi\right)^{2}\right]\right\}
\end{aligned}
$$

while the potential energy of the system will be:

$$
\begin{aligned}
E_{p o t} & =\left[M z_{M}+2 m\left(z_{M}+r \sin \phi\right)\right] g \\
& =(2 m+M) g z_{M}+2 m g r \sin \phi
\end{aligned}
$$

Using (5) and (6), we can write the Lagrangian $L$ of the dynamic system as follows:

$$
\begin{aligned}
L= & T-V \equiv E_{k i n}-E_{p o t} \\
= & \frac{1}{2}\left[M\left(\dot{x}_{M}^{2}+\dot{z}_{M}^{2}\right)+m\left(\dot{x}_{M}-r \omega \sin \phi\right)^{2}\right. \\
& \left.+m\left(\dot{x}_{M}+r \omega \sin \phi\right)^{2}+2 m\left(\dot{z}_{M}+r \omega \cos \phi\right)^{2}\right] \\
& -\left[(2 m+M) g z_{M}+2 m g r \sin \phi\right]
\end{aligned}
$$

The two generalized coordinates of the dynamic system may be chosen as follows:

$$
q_{1}=x_{M}(t), \quad q_{2}=z_{M}(t)
$$

By virtue of (8) and considering $\phi=\phi(t)$ while all four quantities $(m, g, r, \omega)$ are constants, (7) becomes:

$$
\begin{aligned}
& L\left(q_{1}, q_{2}, \dot{q}_{1}, \dot{q}_{2}\right)=\frac{1}{2}\left[M\left(\dot{q}_{1}^{2}+\dot{q}_{2}^{2}\right)+m\left(\dot{q}_{1}-r \omega \sin \phi\right)^{2}\right. \\
& \left.+m\left(\dot{q}_{1}+r \omega \sin \phi\right)^{2}+2 m\left(\dot{q}_{2}+r \omega \cos \phi\right)^{2}\right] \\
& -\left[(2 m+M) g q_{2}+2 m g r \sin \phi\right]
\end{aligned}
$$

Following Reference [6], the equation of motion is:

$$
\frac{d}{d t}\left(\frac{\partial L}{\partial \dot{q}_{i}}\right)-\frac{\partial L}{\partial q_{i}}=0 \text { for } i=1,2
$$

For $i=1, \operatorname{Eq}(10)$ implies that:

$$
\ddot{q}_{1}=0 \text {, }
$$

which means that the object does not accelerate and therefore preserves its initial (zero) horizontal velocity, i.e. $x_{M}(t) \equiv 0$, as anticipated due to the symmetry.

For $i=2$, after manipulation, $\operatorname{Eq}(10)$ becomes:

$$
(2 m+M) \ddot{z}_{M}+2 m r(\sin \phi)^{\bullet \bullet}+(2 m+M) g=0
$$

Considering that at the initial time $t=0$ the angular velocity and the angular position are $\omega_{0}$ and $\phi_{0}$, respectively, the exact solution of $\mathrm{Eq}(12)$ is obtained as follows:

$$
\begin{aligned}
z_{M}(t)= & \left(z_{0}+v_{0} t-1 / 2 g t^{2}\right)+\left(\mu \omega_{0} \cos \phi_{0}\right) t \\
& -\mu\left(\sin \phi-\sin \phi_{0}\right)
\end{aligned}
$$

and

$$
\dot{z}_{M}(t)=\left(v_{0}-g t\right)-\mu\left(\omega \cos \phi-\omega_{0} \cos \phi_{0}\right)
$$

where

$$
\mu=2 m r /(2 m+M)
$$

It should become clear that equations (12)-(14) are valid either the angular velocity is variable or constant.

We note that $\mathrm{Eq}(13)$ consists of the usual term that appears in the accelerated motion, plus the term $\left(\mu \omega_{0} \cos \phi_{0}\right) t$, which is proportional to time, accompanied by a minor sinusoidal term.

Equating the sum of initial kinetic and potential energy, $\mathrm{Eq}(5)$ and $\mathrm{Eq}(6)$, with the potential energy at the upper point, that is $(2 m+M) g z_{\max }$, we can approximately write that

$$
z_{\max } \cong \frac{\left(\mu \omega_{0} \cos \phi_{0}\right)^{2}}{2 g}
$$

Also, the time required for the mechanism to reach the abovementioned upper point is obtained by setting the object velocity equal to zero. Thus, ignoring the term $-\mu \omega \cos \phi$ in $\mathrm{Eq}(14)$, we can approximately write that

$$
t_{\max } \cong \frac{\mu \omega_{0} \cos \phi_{0}}{g}
$$


In other words, it is like as if the object performed a straight line motion with constant acceleration, $-g$, and upward initial velocity $v_{0}=\mu \omega_{0} \cos \phi_{0}$. This means that the critical quantity to determine the upper point of object's orbit is the initial polar angle $\phi_{0}$. Then, the altitude becomes maximum when the eccentric masses are initially on the same horizontal line with the centroid of the object $\left(\cos \phi_{0}=1\right)$, and at the same time no external (support) forces exist.

\subsubsection{Energy Conservation}

Let us suppose that the motor stops feeding the rotation of the rods and, at the same time, the object is left to fall. After considerable manipulation, the condition that the sum of kinetic and potential energy -given by equations (5) and (6) - is preserved to be constant, leads to the following relationship for the variation of the angular velocity:

$$
\omega(t)=\omega_{0} \sqrt{\frac{2 m \sin ^{2} \phi_{0}+M}{2 m \sin ^{2} \phi(t)+M}}
$$

Considering that $\omega(t)=\dot{\phi}(t), \quad \mathrm{Eq}(18)$ can be numerically solved using, for example, the Runge-Kutta method, as implemented by ode45 in MATLAB®.

\subsubsection{Internal Forces and Moments}

If we consider half of the mechanism, we can calculate the force and moment transmitted by one half to the other one.

\subsubsection{Forces}

Concerning forces, it is easily found that the two components of the force exerted from the left to the right half will be:

$$
\begin{aligned}
F_{\text {int }, X} & =(M / 2) \ddot{x}_{M}+m \ddot{x}_{m} \\
& =-m r\left(\ddot{\phi} \sin \phi+\dot{\phi}^{2} \cos \phi\right)
\end{aligned}
$$

and

$$
F_{\mathrm{int}, Z}=(M / 2) \ddot{z}_{M}+m \ddot{z}_{m}+(M / 2+m) g \equiv 0
$$

The sign of $F_{\text {int } X}$ refers to the positive or negative direction of the force component and is not related whether tension or compression occurs. In fact, a positive $F_{\text {int } X}$ corresponds to compression whereas a negative to tension.

\subsubsection{Moments}

Concerning moments, the bending moment in the middle of the mass $M$ is obtained considering the elementary equation that the total moment of external forces with respect to the center of mass for the half mechanism equals to the rate of total angular momentum, that is $\overrightarrow{\mathbf{M}}_{b}=d \overrightarrow{\mathbf{L}} / d t$.

In more details, with respect to the center of mass $\mathrm{C}$ for the half mechanism the angular momentum is given in terms of the position vectors for the center of mass $C_{1}$ of half the object mass (i.e. $M / 2$ ) and the corresponding eccentric mass $m$, by:

$$
\overrightarrow{\mathbf{L}}=\overrightarrow{\mathbf{r}}_{r e l, 1} \times(M / 2) \overrightarrow{\mathbf{v}}_{M}+\overrightarrow{\mathbf{r}}_{r e l, 2} \times(m) \overrightarrow{\mathbf{v}}_{m}
$$

whence (see Figure 2)

$$
\begin{aligned}
\overrightarrow{\mathbf{M}}_{b} & =\frac{d \overrightarrow{\mathbf{L}}}{d t} \\
= & \frac{d \overrightarrow{\mathbf{r}}_{r l, 1}}{d t} \times(M / 2) \overrightarrow{\mathbf{v}}_{M}+\overrightarrow{\mathbf{r}}_{r e l, 1} \times(M / 2) \frac{d \overrightarrow{\mathbf{v}}_{M}}{d t} \\
& +\frac{d \overrightarrow{\mathbf{r}}_{r e l, 2}}{d t} \times(m) \overrightarrow{\mathbf{v}}_{m}+\overrightarrow{\mathbf{r}}_{r e l, 2} \times(m) \frac{d \overrightarrow{\mathbf{v}}_{m}}{d t}
\end{aligned}
$$

Substituting in $\operatorname{Eq}(22)$ the relative vectors by $\overrightarrow{\mathbf{r}}_{r e l, 1}=\overrightarrow{\mathbf{r}}_{1}-\overrightarrow{\mathbf{r}}_{C}$ and $\overrightarrow{\mathbf{r}}_{r e l, 2}=\overrightarrow{\mathbf{r}}_{2}-\overrightarrow{\mathbf{r}}_{C}$, that is in terms of the position vectors $\left(\overrightarrow{\mathbf{r}}_{1}\right.$ and $\overrightarrow{\mathbf{r}}_{2}$ ) with respect to the axis origin $\mathrm{O}$ (along the vertical axis of symmetry), and considering that the position $\overrightarrow{\mathbf{r}}_{C}$ of the center of mass for the right half mechanism is given by the well known expression $\overrightarrow{\mathbf{r}}_{C}=(M / 2) \overrightarrow{\mathbf{r}}_{1}+m \overrightarrow{\mathbf{r}}_{2} /(M / 2+m)$, after manipulation the bending moment is finally given by the formula:

$$
\overrightarrow{\mathbf{M}}_{b}=\frac{(M / 2) m}{(M / 2+m)} \cdot\left(\overrightarrow{\mathbf{r}}_{2}-\overrightarrow{\mathbf{r}}_{1}\right) \times\left(\overrightarrow{\mathbf{a}}_{2}-\overrightarrow{\mathbf{a}}_{1}\right)
$$

Since the vector $\left(\overrightarrow{\mathbf{r}}_{2}-\overrightarrow{\mathbf{r}}_{1}\right)$ in $\operatorname{Eq}(23)$ equals to $\left(\vec{E} C_{1}\right)$, it becomes obvious that it depends not only on the position $L_{1}$ of the center $\mathrm{C}_{1}$ but also on the position $L_{2}$ of the attachment point $\mathrm{P}$ of the rod on the object.

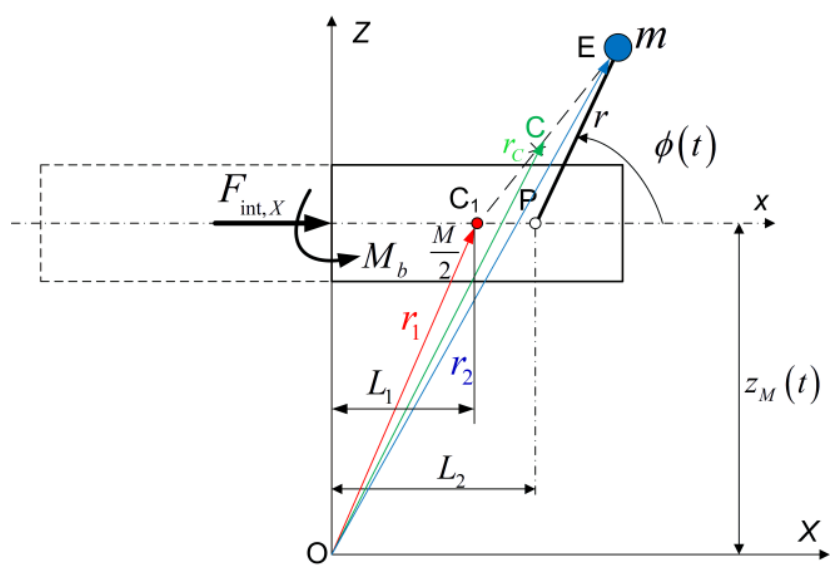

Figure 2. The free body diagram for the right half of "Dean drive", to determine internal loads

\subsection{Single eccentricity}

\subsubsection{General Conditions}

In case that the system consists of only one eccentric mass $m$ (attached to point E), which is connected to the object $M$ through the rigid rod EP (Figure 3), mechanics becomes quite different. The motion depends mainly on (i) whether the point $\mathrm{P}$ coincides with the center of mass of the object or not, and also on (ii) the type of joint at P.

In general, the system of the rigid rod EP associated with the masses $(m, M)$ is a closed system except of the gravitational forces. Therefore, with respect to its center of mass $(\mathrm{C})$ to which the moment of gravity forces vanishes, the angular momentum is preserved. Obviously, the aforementioned center of mass may be firmly connected to the rod EP (when P is the centroid of the object) or may also vary (when $\mathrm{P}$ is not the previously mentioned centroid). 


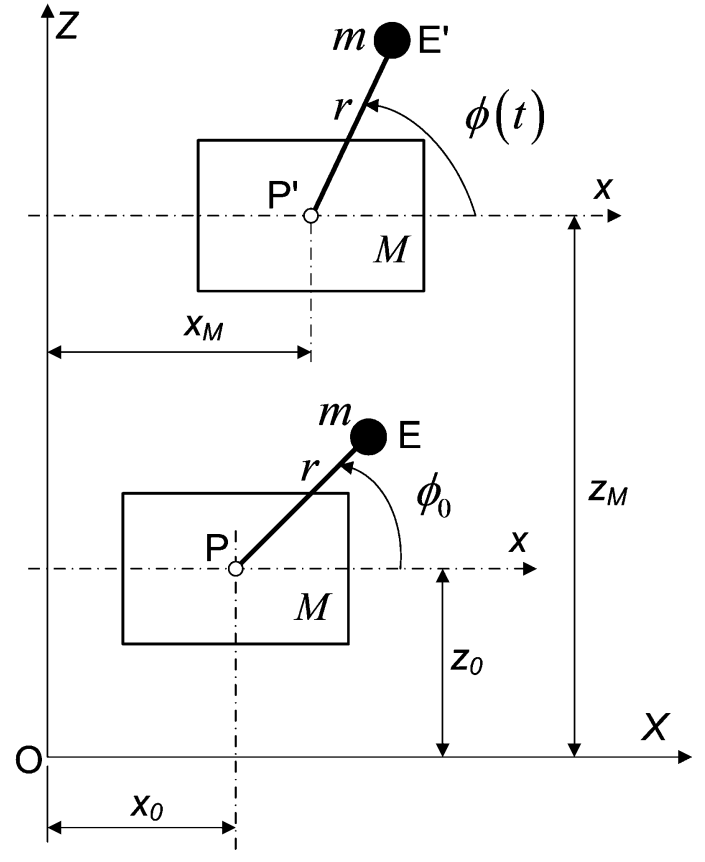

Figure 3. A single eccentric mass attached to the center of mass of the object (of mass $M$ ) moves horizontally and vertically from the initial position $\mathrm{P}$ to the current $\mathrm{P}^{\prime}$

In the particular case that (a) the point $\mathrm{P}$ actually coincides with the center of mass of the object and (b) considering a proper (e.g. ball or hinge) joint between the object and the connecting rod, the internal torque between the object and the connecting rod EP vanishes at $\mathrm{P}\left(M_{y}=\right.$ 0 ), and therefore the free diagram of the object ( $L=I \dot{\omega})$ leads to the fact that the object maintains its initial angular momentum. Without loss of generality, we assume that initially the object $\mathrm{P}$ does not rotate $(\omega=0)$ and therefore it continues not rotating. Under these conditions, the rigid rod EP rotates as if two lumped masses were attached to the points $\mathrm{E}$ and $\mathrm{P}$. In more details, the center of mass $\mathrm{C}$ of the two-body system $(\mathrm{m}$, $M)$ lies always along the segment EP at a distance $r_{E}=$ $(\mathrm{CE})$ and $r_{P}=(\mathrm{CP})$, given by:

$$
r_{E}=\frac{M}{m+M} \cdot r, r_{P}=\frac{m}{m+M} \cdot r
$$

Considering again the entire system $(m, M)$, the total angular momentum wrt $\mathrm{C}$ is given by the formula:

$$
L_{\text {total }}=I_{C M}{ }^{\omega_{C M}}
$$

where

$$
I_{C M}=M r_{P}^{2}+m r_{E}^{2}
$$

Substituting $\mathrm{Eq}(24)$ into $\mathrm{Eq}(26)$, one obtains:

$$
I_{C M}=\frac{M m}{(M+m)} \cdot r^{2}
$$

\subsubsection{Equations of Motion}

Although the previous subsection (2.2.1) could be the starting point to derive the equations of motion, in order to be consistent with subsection 2.1 we follow the Lagrangian formulation as well.

Therefore, in case of an object with mass $M$ and an eccentric mass $m$, the kinetic energy of the system will be:

$$
\begin{aligned}
& E_{\text {kin }}=\frac{1}{2}\left[M\left(\dot{x}_{M}^{2}+\dot{z}_{M}^{2}\right)+m\left(\dot{x}_{m}^{2}+\dot{z}_{m}^{2}\right)\right] \\
& =\frac{1}{2}\left\{M\left(\dot{x}_{M}^{2}+\dot{z}_{M}^{2}\right)+m\left[\left(\dot{x}_{M}-r \omega \sin \phi\right)^{2}+\left(\dot{z}_{M}+r \omega \cos \phi\right)^{2}\right]\right\}
\end{aligned}
$$

while the potential energy of the system will be:

$$
\begin{aligned}
E_{p o t} & =\left[M z_{M}+m\left(z_{M}+r \sin \phi\right)\right] g \\
& =(m+M) g z_{M}+m g r \sin \phi
\end{aligned}
$$

Taking the same generalized variables as in $\operatorname{Eq}(8)$, Lagrange equations $(\mathrm{Eq}(10))$ lead to the following system of decoupled ODEs:

$$
(m+M) \ddot{x}_{M}+m r(\cos \phi)^{\bullet \cdot}=0
$$

and

$$
(m+M) \ddot{z}_{M}+m r(\sin \phi)^{\bullet \bullet}+(m+M) g=0
$$

Since the angular velocity $\omega$ is a constant, the above ODEs are easily integrated in time $t$, progressively obtaining for the horizontal motion:

$$
\dot{x}_{M}(t)=u_{0}-\hat{\mu} \omega\left(\sin \phi-\sin \phi_{0}\right),
$$

and

$x_{M}(t)=x_{0}+u_{0} t-\left(\hat{\mu} \omega \sin \phi_{0}\right) t-\hat{\mu}\left(\cos \phi-\cos \phi_{0}\right)$

while for the vertical motion:

$$
\dot{z}_{M}(t)=\left(v_{0}-g t\right)-\hat{\mu} \omega\left(\cos \phi-\cos \phi_{0}\right),
$$

and

$$
\begin{aligned}
z_{M}(t) & =\left(z_{0}+v_{0} t-1 / 2 g t^{2}\right) \\
& +\left(\hat{\mu} \omega \cos \phi_{0}\right) t \\
& -\hat{\mu}\left(\sin \phi-\sin \phi_{0}\right)
\end{aligned}
$$

where

$$
\hat{\mu}=\frac{m r}{(M+m)}
$$

and $\phi=\phi_{0}+\omega t$.

\subsubsection{Energy Conservation}

Based on the closed form expressions of the four velocity components $\left(\dot{x}_{p}, \dot{z}_{p}, \dot{x}_{e}, \dot{z}_{e}\right)$, after manipulation the total mechanical energy is found always to be constant and therefore equal to its initial value:

$$
E_{\text {total }}=1 / 2 m_{e} r\left(r \omega^{2}+2 g \sin \phi_{0}\right)
$$

\section{Numerical Results}

\subsection{Example 1: Twin Eccentrics}

Following [5], we consider a contra-rotating mechanism with the following data:

-Rotating mass $\quad: m=1 \cdot 0 \mathrm{~kg}$ (wrt. Eq(12)-(15))

- Mass of the object B : $M=5 \cdot 0 \mathrm{~kg}$

- Radius of rigid rod $: r=0 \cdot 10 \mathrm{~m}$ 
- Angular velocity $\quad: \omega=314 \cdot 16 \mathrm{~s}^{-1}(3000 \mathrm{rpm})$

- Initial polar angle of the rods: $\phi_{0}=0 \mathrm{deg}$

- Acceleration of gravity: $g=9 \cdot 81 \mathrm{~m} / \mathrm{s}^{2}$

In this particular case where the anti-clockwise angular velocity is ensured to be constant by the aid of a motor, the result of the kinematical simulation is shown in Figure 4. The graph reminds the vertical shoot of a projectile, well known from lyceum physics. As it has been previously shown [5], the maximum altitude of the object is achieved when the object is left free to fall at the time where the two rigid rods have taken an ideally horizontal position, that is $\phi_{0}=0$.
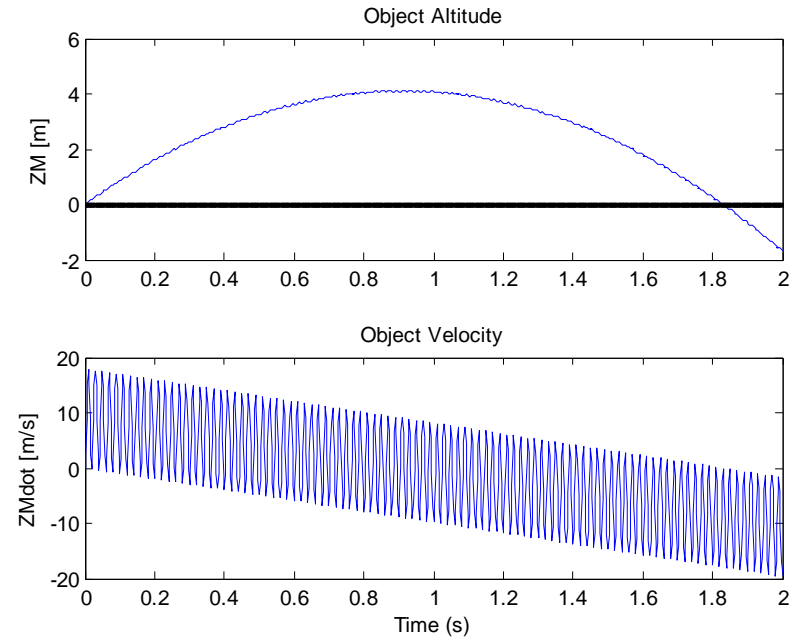

Figure 4. The calculated motion of the object (top: the altitude versus time, bottom: the object velocity)

Moreover, the variation of forces and moments in the middle of the symmetric object (at the axis of symmetry) is shown, for the first time, in Figure 5.
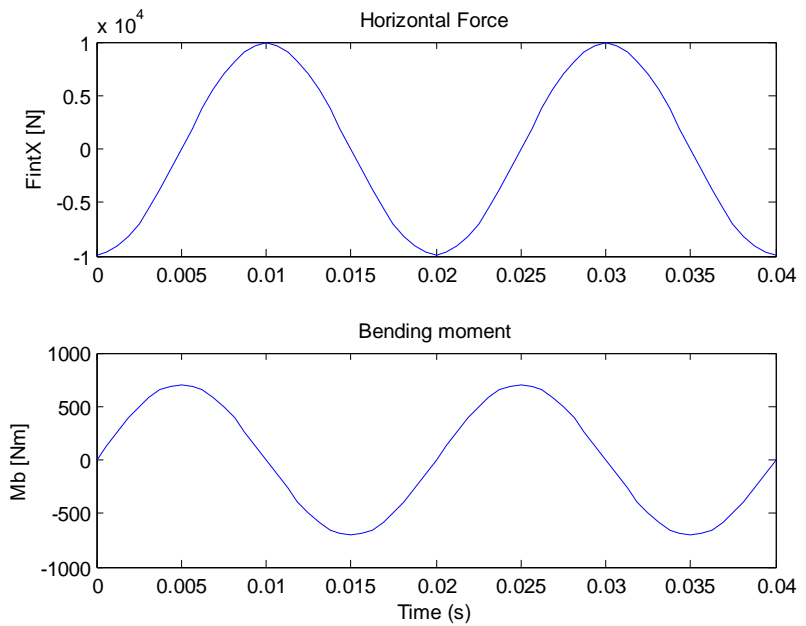

Figure 5. The calculated loads in the middle of the object (top: axial force, bottom: bending moment)

\subsection{Example 2: Single Eccentric Mass}

We select very similar data with those in Example 1:

- Rotating mass $\quad: m=2 \cdot 0 \mathrm{~kg}$ (wrt. Eq(36))

- Mass of the object B : $M=5 \cdot 0 \mathrm{~kg}$

- Radius of rigid rod $: r=0 \cdot 10 \mathrm{~m}$

- Angular velocity $: \omega=314 \cdot 16 \mathrm{~s}^{-1}(3000 \mathrm{rpm})$

- Initial polar angle of the rods: $\phi_{0}=0, \ldots, 360 \mathrm{deg}$

- Acceleration of gravity: $g=9 \cdot 81 \mathrm{~m} / \mathrm{s}^{2}$
It is clarified that here the rotating mass was obtained equal to $2 \mathrm{~kg}$, which equals to the total mass of both masses used in Example 1. Therefore, Equations (30,31) are valid for $m=2 \mathrm{~kg}$, while Eq (12) is valid for $m=1 \mathrm{~kg}$.

Again for anti-clockwise rotation, the results are shown in Figure 6, where one can notice that the track of the object highly depends on the initial value of the polar angle $\phi_{0}$, as it was also the case in Example 1. In more details, when $-180 \leq \phi_{0} \leq 0$ deg (equivalently $180 \leq \phi_{0} \leq 360 \mathrm{deg}$ ), the object moves from the left to the right, whereas when $0<\phi_{0}<180 \mathrm{deg}$ it moves from the right to the left (not shown in the graph).

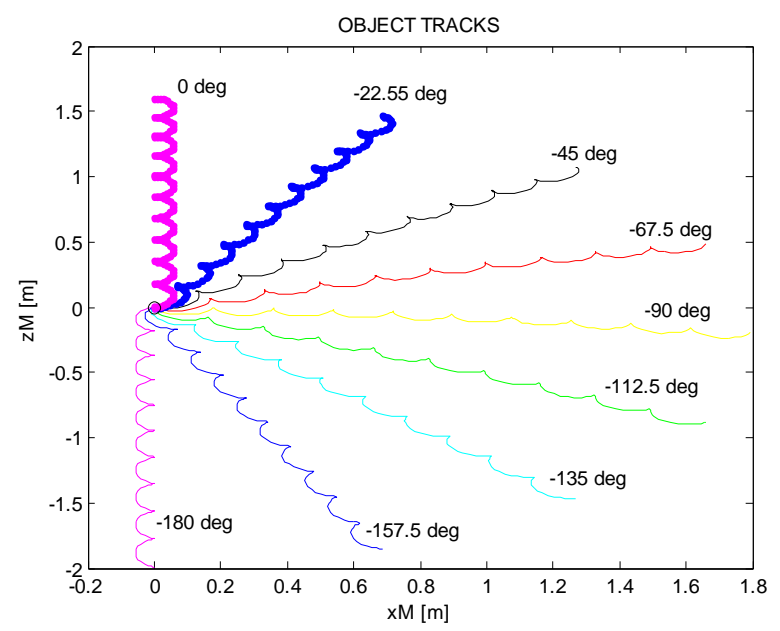

Figure 6. The track followed by the center of mass of the object, for the same anti-clockwise angular velocity and several initial polar angles $\phi_{0}=-180, \ldots, 0$ degrees.

Moreover, as shown in Figure 7, the maximum altitude is obtained when $\phi_{0}=0$, and is identical with that of the contra-rotating mechanism (cf. Eq ((13) with Eq (35)).

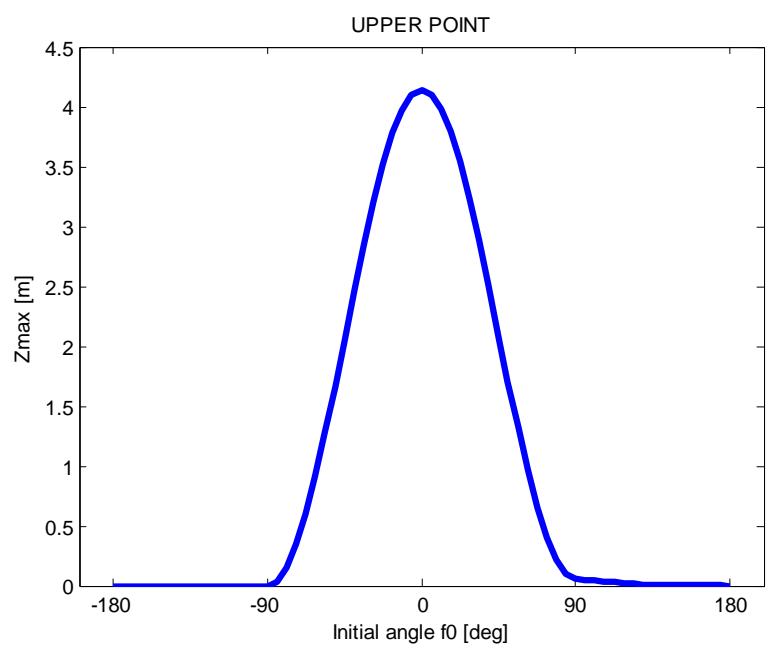

Figure 7. The maximum altitude of the object achieved for the same anti-clockwise angular velocity and several initial polar angles $\phi_{0}=-180, \ldots, 0$ degrees

Finally, in Figure 8 we compare all possible cases concerning Example 1 and Example 2. For almost 50 periods in rotation of the eccentric masses, the numerical solutions for both constant (achieved by the motor in Example 1, and without any motor in Example 2) as well as variable angular velocities are very close one another. 
In other words, either the motor is switched on (and controlled so as to preserve the angular velocity of the eccentrics) or the motor is switched off (and the mechanical energy is preserved thus demanding a variable angular velocity, cf. Eq (18)), the maximum possible altitude the object can reach is the same. Again, the only difference between contra-rotation and single eccentric mass mechanisms is only the horizontal motion of the object, which is possible only for the single mechanism, of course under the conditions illustrated in Figure 6.

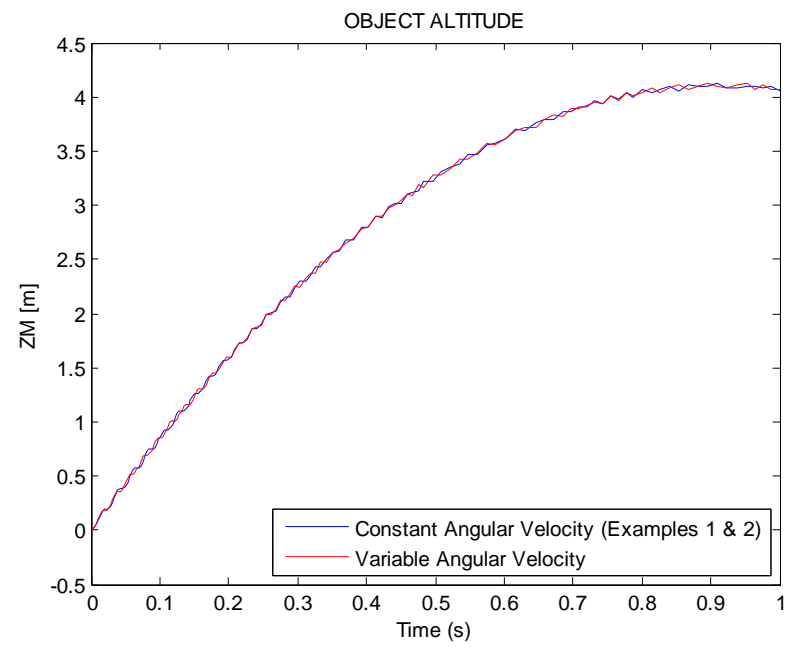

Figure 8. Comparison between Example-1 and Example-2 concerning the track followed in three cases

\subsection{Example 3: Simplified Hydrogen Molecule or Hydrogen Atom}

This example is of rather academic (educational) importance, in the sense that it only depicts the great role of the initial angular momentum in the motion of the object, the latter taken to be the proton of a hydrogen atom (1836 times heavier than the rotating electron [8]). It has been chosen because the two previous examples (Example-1 and Example-2) have already prepared the reader to understand the role of one or two eccentric masses, which could correspond to the hydrogen molecule (two rotating electrons) or hydrogen atom (one rotating electron), respectively.

For purposes of completeness, we remind that when two hydrogen molecules approach, the forces consist of three parts: exchange, quadrupole, and dispersion (van der Waals) forces [7]. These intermolecular forces are very important because they achieve to keep the molecules in close distance from their neighboring ones and not escape; otherwise, under certain individual circumstances, incredible phenomena could happen.

\subsubsection{Virtual Hydrogen Molecule}

In order to create similar conditions with those in Example 1, we assume that the intermolecular forces suddenly vanish exactly when both atoms in the molecule are on their horizontal position (Figure 9, bottom).

In order to estimate the maximum altitude which the hydrogen atom or molecule can reach, it becomes necessary to calculate the mass ratio $\mu$ (i.e. the unknown radius $r$ ) and also the angular velocity $\omega$. The easiest way to achieve it is to consider the old Rutherford-Bohr model of hydrogen atom.
The tangential velocity, $v=\omega r$, is calculated in terms of classical mechanics by equating the centripetal force with the Coulomb force, that is:

$$
\frac{m_{e} v^{2}}{r}=\frac{k_{e} e^{2}}{r^{2}},
$$

where $m_{\mathrm{e}}$ is the electron's mass, $e$ is the charge of the electron, and $k_{e}=1 / 4 \pi \varepsilon_{0}$ is Coulomb's constant ( $\varepsilon_{0}$ is the permittivity constant). This equation determines the electron's speed at any radius $r$ :

$$
v=\sqrt{\frac{k_{e} e^{2}}{m_{e} r}}
$$

In addition, we consider the quantum rule, according to which the angular momentum $L=m_{\mathrm{e}} v r$ is an integer multiple of $\hbar(=h / 2 \pi)[8]$ :

$$
m_{e} v r=n \hbar, n=1,2,3, \ldots
$$

Substituting (39) into (40) gives an equation for $r$ in terms of $n$ :

$$
\sqrt{k_{e} e^{2} m_{e} r}=n \hbar
$$

so that the allowed orbit radius at any $n$ is:

$$
r_{n}=\frac{n^{2} \hbar^{2}}{k_{e} e^{2} m_{e}}
$$

The smallest possible value of $r$ in the hydrogen atom, for $n=1$, is called the Bohr radius.

For the purposes of this paper we assume that the motion of every electron takes place on the vertical plane and the distance between the two protons (nuclei) in the virtual hydrogen molecule is constant, thus the two nuclei compose the "object" of mass $M$ for which our theory (section 2.1) has been developed. As previously was explained, each electron corresponds to the eccentric mass $m$. According to $\mathrm{Eq}(42)$ the distance between every electron and its corresponding proton (nucleus) is constant and transfers axial forces.

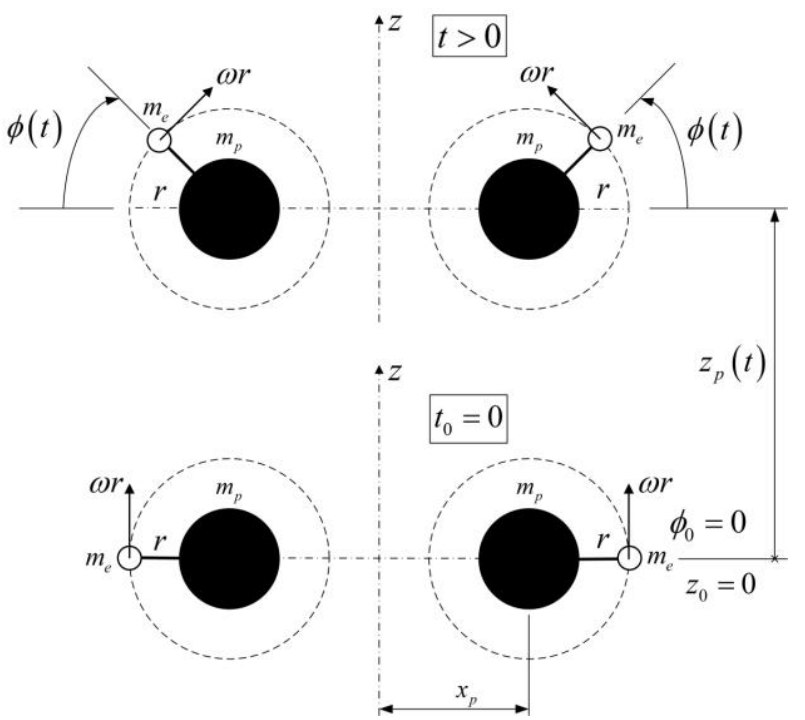

Figure 9. A virtual set up of a synchronized hydrogen molecule moving upwards (bottom figure refers to the initial position, $\mathrm{t}_{0}=0$, where both electrons are assumed at the horizontal level) 
We consider the following well established data:

$$
\begin{array}{ll}
\text { - } \hbar=6.63 \times 10^{-34} /(2 \pi) & \text { [joule.sec }] \\
\text { - } \varepsilon_{0}=8.85 \times 10^{-12} & {[\text { farad } / \mathrm{m}], k_{e}=1 / 4 \pi \varepsilon_{0}} \\
\text { - } e=-1.602 \times 10^{-19} & {[\text { Coulomb }]} \\
\text { - } m_{e}=9.109 \times 10^{-31} & {[\mathrm{~kg}]} \\
\text { - } m_{p}=1.672 \times 10^{-27} & {[\mathrm{~kg}]} \\
\text { - } \mathrm{g}=9.81 & {\left[\mathrm{~m} / \mathrm{s}^{2}\right]}
\end{array}
$$

Then the Bohr radius is equal to:

$$
r_{1}=\frac{\hbar^{2}}{k_{e} e^{2} m_{e}} \approx 5.29 \times 10^{-11} \mathrm{~m}
$$

Moreover, Eq (39) implies that the electron velocity is calculated equal to:

$$
v=2.1869 \times 10^{6} \mathrm{~m} / \mathrm{s}
$$

whereas its angular velocity $(\omega=v / r)$ is:

$$
\omega=4.1287 \times 10^{16} \mathrm{~s}^{-1} \text {. }
$$

Also, Eq (16) and Eq (17) imply that:

$$
z_{\max }=7.2273 \times 10^{4} \mathrm{~m} @ 72 \mathrm{~km}
$$

and

$$
t_{\text {max }}=121.39 \mathrm{~s} .
$$

Although there is no motor to maintain a constant angular velocity $\omega$ (as happened in Example 1), we assume for instance that the latter is practically constant and then we intend to come back to check the validity of this assumption. Under this assumption, the number of revolutions until the molecule reaches the upper point is estimated by:

$$
\frac{\omega t_{\max }}{2 \pi}=7.9763 \times 10^{17} \text { revolutions }
$$

Since the molecule moves upward, a part of the kinetic energy is transformed into gravitational potential energy $2\left(m_{p}+m_{e}\right) g z_{\max }$, a fact that somehow reduces the angular velocity. Actually, for the current Rutherford-Bohr model the initial kinetic energy $K_{0}=2 \cdot\left(1 / 2 m_{e} v^{2}\right)$ equals to $4.3566 \times 10^{-18}$ Joules, while the potential energy at the altitude of $72 \mathrm{~km}$ is only $2.3722 \times 10^{-21}$ Joules. As the latter corresponds to a very small relative variation of the kinetic energy, only $0.05 \%$ with respect to $K_{0}$, the angular velocity of the electron does not practically change.

Applying the above model for more harmonics, $n$, the results are shown in Table 1.

Table 1. Maximum altitude for several harmonics (Initial condition: $\phi_{0}=0$ )

\begin{tabular}{|c|c|c|}
\hline $\begin{array}{c}\text { Harmonic } \\
(n)\end{array}$ & $\begin{array}{c}\text { Maximum Altitude } \\
z_{\max }(\mathrm{km})\end{array}$ & $\begin{array}{c}\text { Rise time } \\
t_{\max }(\mathrm{s})\end{array}$ \\
\hline 1 & 72.3 & 121.4 \\
\hline 2 & 18.1 & 60.7 \\
\hline 3 & 8.0 & 40.5 \\
\hline 4 & 4.5 & 30.3 \\
\hline 5 & 2.9 & 24.3 \\
\hline
\end{tabular}

In all cases, the variation of the angular velocity $\omega$ is less than 0.03 percent with respect to its initial value.

Obviously, for any different initial condition, $\phi_{0}$, the results of Table 1 must be properly multiplied by either $\cos ^{2} \phi_{0}$ or $\cos \phi_{0}$, according to $\operatorname{Eq}(16)$ or $\operatorname{Eq}(17)$, respectively.

\subsubsection{Virtual Hydrogen Atom}

If now the hydrogen atom is isolated from its molecule, the theory of Section 2.2 (single eccentricity) and the closely related Example 2 are applicable. Due to identitity between $\operatorname{Eq}(13)$ and $\operatorname{Eq}(35)$, the maximum altitude for hydrogen atom is the same with that the hydrogen molecule can reach. Therefore, the results of Table 1 are applicable to the hydrogen atom as well, for the additional reason that the angular velocity is preserved per se. Concerning the horizontal motion, except of the case $\phi_{0}= \pm 180$ and 0 degrees, in all other cases it is endless (cf. Figure 6).

\section{Discussion}

Concerning the twin contra-rotating mechanism, the graphs of internal force (alternating between tension and compression) and alternating internal bending moment was presented for the first time. The shear forces along the axis of symmetry of the object were found to be zero, a fact that is theoretically supported by the perfect symmetry in mechanism in both shape and external loading.

Concerning the mechanism based on one single eccentric mass only, it was made clear that the main reason that the angular velocity is constant is the lack of external moment on the object. This study was restricted to the case that the point of attachment coincides with the center of mass $\mathrm{C}_{1}$ of the object. If the latter does not hold, the moment of object's weight with respect to $C_{1}$ will induce an additional angular momentum that has to be taken into account in the formation of modified equations, which are not included in this work. A possible application of this theory is the biomechanics of long jump.

Concerning the hydrogen molecule model, it is a calculation of classical motion of a hydrogen molecule when the intermolecular force between it and nearby hydrogen molecules suddenly disappears. The calculation assumes an unlikely initial configuration for the electrons around the two nuclei: as far away as possible from each other in a Bohr orbital with opposite angular momentum so that each velocity is upward. The paper continues to make assumptions that are more based on convenience than the physics of the situation: a Bohr model of each electron around the proton (but still bound as a hydrogen molecule), a constant angular velocity of the electrons even though the initial momentum of the electron is what propels the molecule upward.

On the other point of view, the Bohr model is only useful for one-electron atoms. In the Hydrogen molecule, there are two electrons interacting and experiencing a nonspherical potential from the two protons. Despite these weaknesses, in the light of Section 2.2 (single eccentric 
mass), for any certain value of the initial polar angle $\phi_{0}$ both the molecule or the atom achieve to reach the same maximum altitude $z_{\max }$. Despite possible reservations from the point of view of modern physicists, and despite the probability that all restrictions made simultaneously occur is practically zero, the educational value of this study is obvious.

In the case we studied, the mass of the proton is much larger, that is about 1836 times the mass of the electron [8]. Nevertheless, the tremendous angular velocity of the electron $\left(2.1869 \times 10^{6} \mathrm{~m} / \mathrm{s}=39.4 \times 10^{6} \mathrm{rpm}\right)$ is capable of producing the motion of the molecule due to its initial momentum.

A secondary shortcoming of our approach is that relevant analysis was based on the assumption of a constant angular velocity. Of course, based on energy conservation, accurate numerical analysis is possible but then the elegance of closed form solution is lost. The fact that the assumption of a constant angular velocity leads to a variation of total energy by $0.05 \%$ (i.e. initial kinetic energy: $K_{0}=4.3566 \times 10^{-18}$ Joules; final gravitational potential energy: $E_{\text {pot }}=2.3722 \times 10^{-21}$ Joules) supports that the analysis of this paper is adequately correct.

Based on the above analytical solution, the linear velocity from $2.1869 \times 10^{6} \mathrm{~m} / \mathrm{s}$ decreases to only $2.1864 \times 10^{6} \mathrm{~m} / \mathrm{s}$, so the variation is only $0.0272 \%$ with respect to the initial value. Since the radius $r$ remains unaltered, the same percentage holds for the angular velocity as well.

Another remark is that at the altitude of $72 \mathrm{~km}$, which corresponds to the smaller (Bohr's) radius, the gravitational acceleration reduces from 9.81 to $9.59 \mathrm{~m} / \mathrm{s}^{2}$, but this is only a variation of approximately 2.2 percent.

In other words, the fully analytical model used in this paper is adequately accurate, at least for the purposes of this work.

It is worth-mentioning that, obviously, the virtual case of an isolated hydrogen molecule is equivalent to the virtual case in which all molecules have the same orientation and also the same initial condition, i.e. at a certain time, $t=0$, all electrons are on their horizontal level with respect to the center of their associated protons. In such perfect synchronization at $t=0$, all vectors of the centrifugal forces lie on the horizontal plane thus no external vertical force is needed to support them.

The topic of the mechanics of contra-rotating oscillating mechanisms has been previously covered in the framework of rigid body dynamics [4,5], whereas alternative figure-eight shaped mechanisms have been proposed (instead of the circumference used in this study) on which the rotating masses move [9]. However, even within the context of contra-rotation, very recent studies have extended the theory by considering elastic waves transmitted through the rigid rods EP that connect the rotating masses with the heavy object [10]. Also a very recent study deals with an "electromagnetic equivalent"
("Tesla" drive), in which no mechanical rotation is necessary [11]. The latter has been said to be promising for the future of aeronautics and astronautics [12].

\section{Conclusion}

It was found that initial angular momentum of eccentric masses, which rotate around heavy objects, force the latter to travel in long distances. The track followed by the objects is primarily proportional to the angular momentum. However, for a specific initial angular momentum, the distance travelled is highly influenced by the initial polar angle at which the mechanism is left free to fall down. In both cases studied, that is (i) contra-rotated and (ii) single mass mechanisms, the object may be initially entirely still but motion is afterwards induced by the momentum of the rotating small masses. In all cases, the motion of the center of mass follows a track similar to that of the well known vertical or oblique shoot for a given initial velocity. The theory was also applied to the hydrogen atom and the hydrogen molecule where intermolecular forces were assumed to vanish and, despite possible concerns from the point of view of modern physics, calculations predicted incredibly large range such as $72 \mathrm{~km}$.

\section{References}

[1] Thompson, R.A., Wehling, M.F. and Evers, J.E., "Evaluation of the haltere as a biologically-inspired inertial rate sensor," AIAA Guidance, Navigation, and Control Conference, Aug. 2008, AFRL-RW-EG-TP-2009-7030.

[2] Blekhman, I.I., Synchronization in Science and Technology, ASME Press, NY, 1988 (in English, translated from Russian 1981).

[3] Dean, N.L., "System for converting rotary motion into unidirectional motion," US Patent 2,886,976, May 19, 1959.

[4] Provatidis, C.G., "Some issues on inertia propulsion mechanisms using two contra-rotating masses," Theory of Mechanisms and Machines, 8 (1). 34-41. 2010.

[5] Provatidis, C.G., "A study of the mechanics of an oscillating mechanism," International Journal of Mechanics, 5 (4). 263-274. 2011.

[6] Goldstein, H., Classical Mechanics, 2nd edn. Addison-Wesley, Reading, 1980.

[7] Margenau, H., "The Forces between Hydrogen Molecules," Physical Review, 64 (5-6). 131-147. 1943.

[8] Halliday, D. and Resnick, R., Physics, Parts I and II, Combined edition, Wiley International Edition, New York, 1966, 1191-1195.

[9] Provatidis, C.G., "A device that can produce net impulse using rotating masses," Engineering, 2 (8). 648-657. 2010.

[10] Provatidis, C.G., "Influence of rotation speed on natural frequency: A short introduction and presentation of an imaginary 'antigravity' world," arXiv: physics.gen-ph/1104.5423. Apr.2011.

[11] Provatidis, C.G. and Gamble M.A., "Support forces in a synchronized rotating spring-mass system and its electromagnetic equivalent," International Journal of Applied Electromagnetics and Mechanics, 41 (3). 313-333. 2013.

[12] Manning, J. "Space, Propulsion \& Energy Sciences International Forum: A Journalist's Notes," Infinite Energy, 103. 18-23. May/June 2012. 